\title{
Experiência de Aprendizagem Móvel: o uso do WhatsApp no Ensino Fundamental
}

\author{
Mixilene Sales Santos Lima ${ }^{1}$, Lavina Lúcia Vieira Lima², Clodomir Silva Lima Neto ${ }^{3}$, \\ José Aires de Castro Filho
}

1 Faculdade Integrada de Araguatins (FAIARA)

77.950-000 - Araguatins - TO - Brazil

2 Prefeitura Municipal de Maracanaú

61939-160 - Maracanaú - CE - Brazil

3 Instituto Federal de Educação, Ciência e Tecnologia do Tocantins (IFTO)

Campus Araguatins - 77.950-000 - Araguatins - TO - Brazil

4 Instituto UFC Virtual - Universidade Federal do Ceará (UFC)

Caixa Postal 15.064 - 91.501-970 - Fortaleza - CE - Brazil

mixilene@gmail.com; clodomir.neto@ifto.edu.br; lavina, aires\}@virtual.ufc.br

Resumo. O presente trabalho apresenta como a aprendizagem móvel, por meio do uso do aplicativo WhatsApp, pode mediar atividades colaborativas entre estudantes participantes de um projeto sobre sustentabilidade, realizado em uma escola da rede pública. Os resultados são apresentados em quatro tópicos (1) autonomia e trabalho em equipe, (2) realização de atividades presenciais e virtuais, (3) diferentes usos dos dispositivos móveis, e (4) atividades colaborativas. $O$ aplicativo possibilitou a realização das atividades colaborativas, além de incentivar os alunos a criar estratégias para o desenvolvimento das atividades de forma autônoma ampliando as possibilidades de aprendizagem.

Abstract. This work shows how mobile learning through the use of WhatsApp application, can mediate collaborative activities among students participating in a project on sustainability, which was done in a public school. The results are presented in four topics: (1) autonomy and teamwork, (2) conducting and virtual activities, (3) different uses of mobile devices, and (4) collaborative activities; The application has enabled the realization of collaborative activities and encouraged students to develop strategies for the development of autonomous activities expanding learning opportunities.

\section{Introdução}

A presença cada vez mais frequente de dispositivos móveis no cotidiano tem modificado a forma como as relações entre as pessoas acontecem e como as informações chegam a elas, de maneira rápida e impactante. $\mathrm{O}$ uso de tablets, laptops e, em especial, smartphones tem crescido visivelmente em todos os ambientes. No ambiente educacional não é diferente: alunos e professores utilizam esse tipo de ferramenta em seu cotidiano escolar para diversas ações, tais como pesquisas, trocas de informações e material didático, por exemplo.

Nesse contexto, temos a chamada Aprendizagem Móvel (ou M-Learning), cujo foco é a possibilidade do uso de dispositivos móveis e suas ferramentas para o desenvolvimento de 
V Congresso Brasileiro de Informática na Educação (CBIE 2016)

Anais dos Workshops do V Congresso Brasileiro de Informática na Educação (CBIE 2016)

atividades de ensino e aprendizagem. Essa aprendizagem se faz não apenas no espaço escolar, mas em todos os espaços sociais. Assim, a mobilidade não é apenas física, mas social.

O presente artigo tem por objetivo apresentar como a Aprendizagem Móvel, por meio do uso do aplicativo WhatsApp, pode mediar atividades colaborativas entre estudantes.

No próximo tópico, serão debatidos os conceitos pertinentes à nossa análise. Em seguida, será apresentada a metodologia do trabalho, o contexto em que se realizou a nossa pesquisa, e como a experiência foi realizada. Por fim, temos os resultados, apresentados em quatro tópicos, e as considerações finais do trabalho.

\section{Aprendizagem móvel}

O uso de diferentes ferramentas para auxiliar os processos de ensino e aprendizagem sempre esteve presente no ambiente escolar. São exemplos de materiais didáticos livros, cadernos, mapas, jornais, entre outros. Assim, a aprendizagem pode ser considerada móvel desde sempre, visto que podemos nos deslocar fisicamente levando esses materiais para qualquer lugar. O termo aprendizagem móvel surge em uma perspectiva recente, com a chegada das tecnologias portáteis, que possibilitam a comunicação e interação por meio de recursos computacionais [Saccol; Schlemmer; Barbosa, 2011]. Com o uso cada vez maior de dispositivos móveis de acesso a Internet, como tablets, smartphones e laptops, ampliam-se também as formas de uso desses recursos para fins educativos, dentro e fora do ambiente escolar.

Os jovens em idade escolar estão entre as faixas etárias que mais utilizam celulares para o acesso a Internet, por exemplo. De acordo com o IBGE, em pesquisa de 2014, 54,1\% da população entre 10 e 14 anos de idade possui aparelho celular ou smartphone e este é o seu principal meio de acesso à internet. No grupo cuja faixa etária é de 15 a 17 anos, esse número aumenta para $80,8 \%$. Além disso, "o celular era um bem pessoal para $93,4 \%$ dos estudantes da rede privada de ensino e para $66,8 \%$ dos da rede pública, (...)"1.

$\mathrm{O}$ uso feito por esses jovens tem como objetivo não apenas o acesso à informação, mas a comunicação e a produção de conteúdos através de aplicativos de mensagem e redes sociais [Saccol; Schlemmer; Barbosa, 2011]. Nesse sentido, entendemos que esse uso é evidenciado como uma possibilidade de interação e colaboração entre os indivíduos em favorecimento à aprendizagem, pois há uma necessidade de ampliar o processo educacional, eliminando as barreiras que impedem a aprendizagem de conteúdos escolares em qualquer lugar, utilizando qualquer recurso que favoreça e proporcione acesso a novos conhecimentos. De acordo com Bulção (2009), essa necessidade deu origem à chamada mobile learning ( $\mathrm{m}$ learning) ou Aprendizagem Móvel.

Para O’Malley et al. (2003, p. 6),a Aprendizagem Móvel é uma modalidade que considera "qualquer tipo de aprendizado que acontece quando o aluno não está em local fixo, ou aprendizagem que acontece quando o aluno aproveita as oportunidades oferecidas pelas tecnologias móveis". Em consonância, Santaella (2013, p. 299) a define "como recursos para educação que podem fazer o aprendizado chegar a quaisquer lugares". Nesse mesmo sentido, a Organização das Nações Unidas para a Educação, a Ciência e a Cultura - UNESCO (2013,

\footnotetext{
1 VILLELA, Flávia. Celular é principal meio de acesso à internet no Brasil, mostra IBGE. 06/04/2016. Disponível em: http://agenciabrasil.ebc.com.br/economia/noticia/2016-04/celular-e-principal-meio-deacesso-internet-na-maioria-dos-lares. Acessado em: $10 \mathrm{ago} / 2016$.
} 
V Congresso Brasileiro de Informática na Educação (CBIE 2016)

Anais dos Workshops do V Congresso Brasileiro de Informática na Educação (CBIE 2016)

p. 8) afirma que "aprendizagem móvel envolve o uso de tecnologias móveis, isoladamente ou em combinação com outras tecnologias de informação e comunicação (TIC), a fim de permitir a aprendizagem a qualquer hora e em qualquer lugar".

Podemos destacar alguns elementos que permeiam e caracterizam a aprendizagem móvel, segundo os autores Sharples; Taylor; e Vavoula (2005), Winter (2006) e Saccol; Schlemmer; e Barbosa (2011):

- Aprendizagem centrada no indivíduo: considerando suas habilidades e conhecimentos, uma vez que o mesmo possui mais autonomia e controle sobre a sua aprendizagem;

- $\quad$ Aprendizagem em contexto: está relacionada à aprendizagem que ocorre além da sala de aula, em contextos diversos, como no trabalho, em casa, em um parque, no ônibus, ou seja, deve-se preocupar em compreender como as pessoas se envolvem em seu ambiente para criar situações de aprendizagem;

- Aprendizagem contínua entre contextos: oferecida pela conectividade, onde o indivíduo pode permanecer conectado e realizando atividades de acordo com o seu deslocamento em um determinado local, bem como pode parar e continuar a realizar algo em contextos diferentes;

- $\quad$ O uso onipresente da tecnologia pessoal e compartilhada: muitas pessoas possuem, no mínimo, um dispositivo móvel, seja celular, smartphone ou laptop. Assim, o indivíduo pode aproveitar as oportunidades de aprendizagem, de acordo com suas necessidades e interesse, sem limites de tempo nem espaço.

Nesse sentido, a aprendizagem pode acontecer em qualquer lugar. Os recursos de aprendizagem estão em determinado local, mas podem ser aplicados ou desenvolvidos em outros lugares. Assim, o indivíduo aprende continuamente, confrontando seus conhecimentos em contextos diferentes. Sharples; Taylor; Vavoula (2005, p. 6) relatam que "a era da tecnologia móvel, pode vir a conceber a educação como uma conversa em seu contexto, ativado pela interação contínua através e com a tecnologia pessoal e móvel". Uma vez que a aprendizagem é facilitada pela mobilidade e conectividade, os dispositivos móveis sem fio se tornam, assim, um apoio à educação.

Winter destaca que os dispositivos móveis são compreendidos como ferramentas mediadoras do processo de aprendizagem, ou seja, a "aprendizagem é mediada por tecnologias móveis, que são em si mesmas entrelaçadas com outras ferramentas de aprendizagem" (2006, p. 7). Além de possuir características específicas, como a mobilidade, portabilidade, interatividade, conectividade, individualidade [Göttsche, 2012, p. 64], o que potencializa e amplia as possibilidades da aprendizagem móvel.

Assim, os dispositivos móveis ampliam as possibilidades de aprendizagem virtual, por oferecerem aos usuários acesso rápido às informações e aos conteúdos em sala de aula, em casa, no trabalho ou em qualquer outro lugar.

Com os dispositivos móveis, cresceu a produção dos aplicativos com diversos fins. Os aplicativos móveis são softwares desenvolvidos para executar funções específicas que funcionem em dispositivos como celulares, smartphones, tablets, entre outros, que proporcionem mobilidade e portabilidade. A área de pesquisa denominada de Computação Móvel tem propiciado e ampliado o aparecimento de inúmeros aplicativos para as tecnologias móveis. Isso demanda suporte à mobilidade e infraestrutura de comunicação sem fio.

Existem aplicativos com diferentes funções como: acesso a informações, às redes sociais; compartilhar informações; assistir e editar vídeos, dentre outros. Todos "estão 
V Congresso Brasileiro de Informática na Educação (CBIE 2016)

Anais dos Workshops do V Congresso Brasileiro de Informática na Educação (CBIE 2016)

associados à interação social, à localização espacial, coleta de dados, rastreamento e muitas outras que poderão ser aplicadas em função da intenção do usuário" [Saboia, et al., 2013, p. 4]. A popularização dos dispositivos móveis (tablets e smartphones) só aumenta, assim como os aplicativos. Segundo a Canaltech Corporate ${ }^{2}$ (2013) destaca que até o ano de 2013 foram produzidos milhares de aplicativos pelas três grandes empresas de tecnologia, Apple, Google e Microsoft.

Nesse universo, cresce também a necessidade de materiais educacionais destinados a esses dispositivos. Aqui no Brasil, autores como Orlandi; Isotani (2012); Mossman; Gomes; Gluz (2012); Rachid; Ishitani (2012); Melo; Carvalho (2014) têm trabalhado na produção de ferramentas de autoria com o intuito de aumentar o número de materiais educacionais, em língua materna, voltados para dispositivos móveis.

Verifica-se que já há a preocupação com o desenvolvimento de aplicativos para educação, mesmo que em pouca quantidade e não muito divulgados. Entretanto, são aplicativos que em sua maioria são para a simples resolução de exercícios. Nessa linha, é importante saber até que ponto a utilização de tecnologias móveis permite ao usuário alcançar resultados que não poderiam ser obtidos sem essas tecnologias [Winter, 2006]. Entretanto, "O grande desafio que se coloca para as escolas e para os educadores é conseguir incluir estas tecnologias de forma produtiva nas suas práticas educativas" [Göttsche, 2012, p. 71].

Nesse sentido, o desenvolvimento de atividades colaborativas também é possibilitado, devido a diferentes aplicativos, nos quais alunos e professores podem elaborar trabalhos a partir de ações síncronas ou assíncronas, interagindo por meio de ferramentas digitais, como na experiência apresentada por Lima; Lima Neto; e Castro Filho (2015).

Entretanto, é necessário refletir sobre a forma de utilizar esses recursos, dispositivos móveis e seus aplicativos, no contexto educacional. Entendemos que uma proposta de aprendizagem colaborativa apoiada por dispositivos móveis demanda acompanhamento intensivo, pois sua utilização precisa ser identificada e analisada, de modo a suscitar o trabalho conjunto dos alunos e professores,

Tal possibilidade, no contexto educacional, potencializa e transforma a sala de aula em um local em que a produção do conhecimento pode acontecer de forma colaborativa, com vários alunos produzindo um único material e aprendendo juntos, permitindo múltipla autoria e o compartilhamento de recursos para além dos limites da escola [Lima, 2015]. Segundo Kasper (2000, p. 25) é "através do esforço da tarefa colaborativa, estruturada que [os alunos] podem atuar como peritos e novatos na mesma tarefa [e] contribuir com blocos de construção para o conhecimento".

Nesse sentido, entendemos que a aprendizagem móvel é potencializada com o uso de aplicativos, que oferecem entretenimentos, informação, possibilidades de compartilhamento, colaboração, e podem ser utilizados para o ensino e a aprendizagem.

Apresentamos, a seguir, o aplicativo utilizado no contexto da experiência realizada.

\subsection{Aplicativo WhatsApp}

O aplicativo WhatsApp não havia sido escolhido para compor os recursos do projeto, mas, por solicitação da turma, foi inserido, já que a maioria possuía um smartphone e tinha acesso

\footnotetext{
${ }^{2}$ Disponível em: <http://corporate.canaltech.com.br/noticia/desenvolvedores/Quanto-os-desenvolvedoresganham-por-cada-aplicativo-criado/>.
} 
V Congresso Brasileiro de Informática na Educação (CBIE 2016)

Anais dos Workshops do V Congresso Brasileiro de Informática na Educação (CBIE 2016)

ao aplicativo. Na página brasileira do WhatsApp ${ }^{3}$ encontra-se a seguinte definição do aplicativo. "WhatsApp Messenger é um aplicativo de mensagens multiplataforma que permite trocar mensagens pelo celular sem pagar por SMS. Está disponível para iPhone, BlackBerry, Android, Windows Phone, e Nokia." Disponíveis também para tablet e mais recentemente para computador, pode ser acessado via WhatsApp $\mathrm{Web}^{4}$.

Além das mensagens básicas, os usuários podem criar grupos de discussão, sem limite de pessoas, inserindo os contados que desejar da sua lista. Permite enviar mensagens ilimitadas, com imagens, vídeos, link, áudio e atualmente faz ligações sem custo de chamadas. Essas funcionalidades o caracterizam como multiplataforma.

Atualmente, observamos que as funcionalidades do aplicativo estão sendo agregadas novas funções, incluindo a anexação de arquivos de diversos formatos.

\section{Metodologia}

Neste trabalho, utilizamos da pesquisa qualitativa por ser uma metodologia que melhor representa os estudos nos aspectos humanos e sociais [Bogdan e Biklen, 1994]. Por sua vez, é segundo Gabre [2012, p.2] "um meio de produção de conhecimento que não busca mensurar, medir, mas, sim, compreender e buscar explicações a valores e significados num meio social".

Apropriamo-nos, ainda, da pesquisa intervenção, por seu caráter de intervenção direta do pesquisador ao ambiente natural dos pesquisados, tendo a proposta de promover mudanças sociais aos pesquisados [Castro; Besset et al, 2008].

A seguir, apresentaremos o contexto em que a pesquisa foi realizada: um projeto desenvolvido em escola da rede pública de Aquiraz-CE, bem como o seu desenvolvimento.

\subsection{Contexto da pesquisa (Planejamento e Participantes)}

A pesquisa foi realizada em uma escola da rede pública, situada no Município de Aquiraz, localizada a 32,30 quilômetros de Fortaleza-CE, com 10 alunos do Ensino Fundamental. As atividades estavam inseridas em um projeto mais amplo, chamado EDigital ${ }^{5}$, que teve como principal objetivo a integração e o trabalho pedagógico com recursos digitais integrados ao currículo escolar com foco em três eixos: formação dos professores; formação de alunos monitores; e realização de projeto com o tema sustentabilidade. Todas as ações do projeto ocorreram no âmbito da escola, a partir de setembro de 2013.

Nesse contexto, propusemos um projeto de intervenção que abordasse a temática sustentabilidade e que fizesse o uso de TDIC e ferramentas da Web 2.0. Contudo, com base nas perspectivas da aprendizagem móvel e aprendizagem colaborativa com apoio computacional, escolhemos os dispositivos móveis, mais precisamente o netbook, por fazer parte do contexto da escola, e os aplicativos de desenho e apresentação de slides da Google, visto que, após as pesquisas, estes foram os que mais apresentaram características favoráveis ao trabalho colaborativo.

\footnotetext{
${ }^{3}$ Disponível em: $<$ https://www.whatsapp.com/>.

${ }^{4}$ Disponível em: $<$ https://web.whatsapp.com/>.

5 A implementação do projeto EDigital deu-se por meio da parceria entre a escola, a Companhia de Energia Elétrica do Estado do Ceará (Coelce) e o grupo de pesquisa Proativa pertencente à UFC. Com isso, a Coelce responsabilizou-se pela infraestrutura tecnológica da escola, contribuindo com 18 netbooks e a disponibilização de rede WiFi para toda a escola. O Grupo Proativa responsabilizou-se pela formação e acompanhamento pedagógico de professores e alunos e realização de projetos com o tema sustentabilidade.
} 
V Congresso Brasileiro de Informática na Educação (CBIE 2016)

Anais dos Workshops do V Congresso Brasileiro de Informática na Educação (CBIE 2016)

Para o trabalho, a escolha dos alunos foi feita por meio de convite e indicação de um professor. O critério de escolha dos participantes foi o interesse dos mesmos e a sua disponibilidade. Assim, participaram do projeto de intervenção dez alunos, com idades entre 12 e 14 anos, de turmas do $7^{\circ}, 8^{\circ}$ e $9^{-0}$ anos do ensino fundamental, do período matutino. Os nomeamos como: Aluno 1, Aluno 2, Aluno 3, Aluno 4, Aluno 5, Aluno 6, Aluno 7, Aluno 8, Aluno 9, Aluno 10.

A seguir detalharemos as atividades do projeto de intervenção, de forma a descrever como se deram todas as ações.

\subsection{Projeto de intervenção - Consciência Sustentável: Caminhando para um Mundo Melhor}

O projeto de intervenção intitulado Consciência Sustentável: Caminhando para um Mundo Melhor, partiu dos temas sustentabilidade e consciência sustentável e dos subtemas Energia, Água e Reciclagem. Os objetivos foram discutir os temas e subtemas; elaborar material digital sobre os mesmos; possibilitar o exercício do papel de cidadão consciente e do trabalho colaborativo.

A intervenção foi desenvolvida, em sua maior parte, no Laboratório de Informática Educativa (LIE) da escola e foi realizado nos meses de setembro e outubro de 2014, em dois encontros semanais de três horas/aula cada, totalizando 14 encontros.

As atividades realizadas nos encontros tinham estrutura previamente fixa e, de modo geral, envolviam contenda (rememória), exibição de vídeos, pesquisa na Internet, discussão em grupo, e produção colaborativa em equipe. Eram planejados pela pesquisadora com antecedência, no entanto, no decorrer das ações, poderiam ser modificadas e ajustadas, de acordo com a necessidade observada no momento ou a partir da sugestão dos alunos.

Os alunos pesquisavam e discutiam sobre o subtema da semana, como, por exemplo, energia sustentável ou reciclagem. Em seguida, elaboravam um desenho digital sobre o assunto, em forma de cartaz ou tirinha. Ao final do projeto, as equipes também elaboraram uma apresentação de slides destacando o que aprenderam, o que realizaram, incluindo seus desenhos e fotos capturadas no transcorrer das atividades. Ao todo, foram feitas 13 produções colaborativas, com um desenho de toda a turma e três desenhos de cada equipe feitos no aplicativo Google Desenho. Já no aplicativo Google Apresentação, cada equipe fez uma sessão.

Cada aluno tinha disponível um netbook com acesso à Internet sem fio, além de realizar atividades com material reciclado e utilizar o smartphone. Estes últimos, acrescidos a partir da interferência da turma, visto que nem sempre as ações planejadas foram efetivadas, e poderiam ser modificadas e/ou adaptadas de acordo com as necessidades surgidas. Tal como as sugestões dos alunos, sempre que possível era exposto o planejamento ao grupo, na tentativa de proporcionar aos alunos a atuação como colaboradores das atividades e fazer sugestões, indicando vídeos, escolhendo os assuntos e como poderiam ser trabalhados.

Além dos dispositivos e aplicativos apresentados, fizemos o uso do aplicativo do WhatsApp, não por planejamento prévio, mas por solicitação dos alunos. Assim, criamos um grupo, intitulado Sustentabilidade, adicionando todos os alunos que possuíam o aplicativo, o qual foi utilizado durante todo o projeto como espaço de socialização de informações e avisos sobre o "curso", como chamado por eles, e que proporcionou momentos de discussão a distância. 
V Congresso Brasileiro de Informática na Educação (CBIE 2016)

Anais dos Workshops do V Congresso Brasileiro de Informática na Educação (CBIE 2016)

\subsection{Coleta de dados}

A coleta de dados foi realizada por meio dos registros dos encontros (diário de campo e conversas no aplicativo WhatsApp), entrevistas realizadas com alguns alunos e materiais digitais produzidos pelos mesmos.

\section{Resultados}

O aplicativo WhatsApp foi adotado como parte das ferramentas utilizadas no projeto, visto que os alunos poderiam manter o contato a distância, em horários além dos encontros, ampliando os espaços e momentos de colaboração. Como Destacam Sacool; Schlemmer e Barbosa (2011, p.7) "aprender em processos de mobilidade [...] implica abrir-se às potencialidades que essas tecnologias oferecem".

Apresentaremos os resultados com foco no uso do aplicativo WhatsApp, embora a pesquisa tenha sido ampliada a outros dispositivos móveis e aplicativos. No entanto, vimos o destaque que o mesmo teve durante o projeto. Uma vez que o uso do aplicativo foi uma interferência dos alunos no desenvolvimento do projeto. Logo no primeiro encontro, os alunos sugeriram utilizar o aplicativo para manter contato entre os componentes do grupo.

Nossa análise será apresentada em tópicos, nos quais descreveremos aspectos que se destacaram durante o desenvolvimento do projeto em que pudemos perceber como a aprendizagem móvel possibilitou e até mesmo favoreceu a realização das atividades colaborativas através do aplicativo utilizado.

\subsection{Autonomia e trabalho em equipe}

O uso de grupo no WhatsApp foi bastante utilizado. Além do grupo da turma em que todos os alunos estavam inseridos, algumas equipes criaram grupos específicos, para facilitar a comunicação entre os membros e o desenvolvimento das atividades, como exemplificado na transcrição abaixo.

Pesquisadora: Mas essa opinião era oral, era lá no recurso ou cada um fazia sua parte...?

Aluno 8: É tinha. Tinha no bate-papo [WhatsApp], tinha um grupo que a gente fez no WhatsApp, tinha é...

Oral mesmo.

Pesquisadora: Esse grupo no bate-papo [WhatsApp] você chegaram a conversar muita coisa sobre as atividades ou não?

Aluno 8: Não! Só algumas coisa pra resolver, tipo antes de vir pra cá. No dia dá, no dia do, no dia de realizar as atividades dos objetos lá de reciclagem, a gente conversou e resolveu essa situação de o que a gente ia fazer,

Pesquisadora: Ah tá. Pelo WhatsApp?

Aluno 8: Pelo WhatsApp, pelo esse grupo.

(Entrevista do Aluno 8)

Vemos assim que o aplicativo possibilitou não somente o acesso à informação (avisos, datas e horários dos encontros e atividades), mas também o desenvolvimento das atividades de forma autônoma, o que pode ser considerado uma forma de envolvimento e comprometimento com o projeto.

\subsection{Realização de atividades presenciais e virtuais}


V Congresso Brasileiro de Informática na Educação (CBIE 2016)

Anais dos Workshops do V Congresso Brasileiro de Informática na Educação (CBIE 2016)

Em diversos momentos, percebemos a utilização de diversas estratégias para o desenvolvimento das atividades por parte, em especial, das equipes, as quais desenvolveram atividades em momentos presenciais e também virtuais. $\mathrm{O}$ aluno 8 (entrevista citada acima) por exemplo, cita uma conversa na qual seu grupo discutia qual material utilizariam e o que fariam com esse material no dia da atividade.Isso evidencia a familiaridade com outras ferramentas de comunicação, usadas inclusive em outros momentos, além dos encontros presenciais da intervenção.

\subsection{Diferentes usos dos dispositivos móveis}

O uso do smartphone foi ampliado a partir de um apelo dos alunos, que sugeriram que o mesmo também fosse usado captura de imagens (no momento de atividades desenvolvidas em campo ou fora de sala de aula), ao invés de utilizarem a câmera dos netbooks. Assim, foi possível demonstrar aos alunos que o aparelho possui funcionalidades passíveis de serem agregadas às atividades educativas. Ao final, socializaram com os componentes de cada equipe as imagens, por meio dos grupos do WhatsApp ou por meio de cabo USB.

A inserção de recursos não previstos inicialmente também é um exemplo de que os alunos tomaram decisões de forma autônoma, em diversos momentos do projeto. Destacamos também que a opção favoreceu a colaboração da sua equipe ou turma, ou seja, serviu para melhorar e facilitar o trabalho do grupo.

\subsection{Atividades colaborativas}

Os aplicativos de Desenho e de Apresentação de Slides do Google foram utilizados em diversos momentos, constituindo, em si mesmo, perfil colaborativo. Os materiais produzidos nos aplicativos eram criados e desenvolvidos em momentos presenciais (nos encontros) e à distância, quando os alunos se comunicavam pelo WhatsApp, combinando um horário para estarem online e realizarem as atividades. Ou seja, os dois aplicativos eram utilizados simultaneamente, no smartphone e no netbook ou computador pessoal.

Enquanto produziam colaborativamente, interagiam utilizando o bate-papo por meio do diálogo escrito e pelo aplicativo WhatsApp com áudios, vejamos.

Aluno 7: Tia, ontem tão legal!A Aluna 9 tava aqui [Escola], no computador [netbook da escola] dela e eu lá em casa, ai ela falou comigo pelo Whatsapp e a gente começou a fazer. A gente, aí eu atrás de descobrir onde é que bota a imagem pra trás, rebolar pra trás pra poder botar duas imagens, descobri.

Pesquisadora: Descobriu?

Aluno 7: Fizemos um bocado de coisa, essas coisas tudim aí, a gente que fez juntas.

Pesquisadora: Eu vi que vocês estavam conversando no bate-papo também.

Aluno 7: Era porque a gente tanto conversava pelo WhatsApp como conversava pelo bate-papo.

Pesquisadora: Que é até mais fácil por que tá no computador [notebook].

Aluno 7: É, fala mais rápido. Só que aí não dava pra mandar, eu também... tem certas coisa que a gente não dá pra escrever, né. A gente manda áudio.

Pesquisadora: Entendi.

(Entrevista do Aluno 7)

Analisamos essas situações a partir do que Sharples; Taylor e Vavoula (2005) falam: o contexto de aprendizagem é construído pelos alunos a partir de suas interações com o meio. 
V Congresso Brasileiro de Informática na Educação (CBIE 2016)

Anais dos Workshops do V Congresso Brasileiro de Informática na Educação (CBIE 2016)

O exemplo mostra como os alunos julgaram os momentos propícios à aprendizagem deles, bem como suas necessidades.

\section{Conclusões}

A aprendizagem se dá no contexto da mobilidade, situada no ambiente real, e é centrada nas necessidades do sujeito, a partir da ação e interação com o meio físico, social e digital, guiada pela observação do entorno, da exploração e da experimentação, realizando aproximações e distanciamentos necessários para a significação.

Entendemos que as tecnologias móveis, permitem que os alunos acessem qualquer tipo de informação, sobre pessoas, locais e lugares que queiram conhecer. Esses alunos, além do acesso rápido, devem fazer uso dessas informações, ressignificá-las e repassá-las a outras pessoas, atribuindo a elas seus conhecimentos.

Nas situações apresentadas, citamos exemplos que caracterizam a aprendizagem móvel sendo explorada pelos alunos, de modo a proporcionar interações e colaborações, seja somente no ambiente escolar, como em outros ambientes. A aprendizagem acontece em dias e horários distintos e em contextos variados.

Concluímos que o conhecimento pode ser construído em uso, ou seja, de forma maleável, possibilitado pela mobilidade e conectividade. Os alunos constroem seus conhecimentos por meio das ações, atos comunicativos e contextos diversos, sem definição de tempo nem espaço. No mais, essas situações sinalizam também como a aprendizagem colaborativa com apoio computacional acontece.

Por fim, acrescentamos que, por não ter sido feito um estudo sistemático sobre sua utilização, recomendamos que outras abordagens foquem especificamente no potencial dessa ferramenta para a colaboração entre os alunos.

\section{Referências}

Bogdan, R.C.; Biklen, S.K. (1994) Investigação qualitativa em educação: uma introdução à teoria e aos métodos. Tradução Maria João Alvarez. Porto: Porto.

Castro, L. R de; Besset, V. L. (Orgs.) (2008) Pesquisa-intervenção na infância e juventude. v.1, Rio de Janeiro: NAU.

Gabre, S. (2012) "Contribuições da pesquisa intervenção na construção de um projeto educativo no museu de arte: pensando a mediação cultural para a pequena Infância”, In: IX ANPED Sul Seminário se pesquisa em educação da região Sul, Caxias do Sul.

Göttsche, Katja. (2012) “Tecnologias móveis: uma mais valia em contextos educacionais?” In: Revista Linhas, Florianópolis, v. 13, n. 2, p. 62-73.

Lima, Mixilene S. S; Lima Neto, C. S.; Castro Filho, J. A. De . (2015) “Aprendizagem móvel no Ensino Fundamental", In: III Workshop sobre Tecnologias Móveis na Educação, Maceió, 2015. p. 825-833.

Lima, Mixilene. S. S (2015). Aprendizagem colaborativa com apoio computacional: uma experiência no ensino fundamental. Dissertação. Mestrado em Educação, Universidade Federal do Ceará.

Lima, Mixilene. S. S.; Osmundo, M. L. F; Mendes, W. M. F.; Nascimento, Karla A. S. Do; Castro Filho, J.A (2014) Trabalho Colaborativo: a produção de aplicativo colaborativo 
V Congresso Brasileiro de Informática na Educação (CBIE 2016)

Anais dos Workshops do V Congresso Brasileiro de Informática na Educação (CBIE 2016)

educacional. In: Francisco de Ari Andrade; Carlos Augusto Viana; Filipe de Menezes Jesuino; Renata Aquino da Silva. (Org.). Educação Brasileira: conceitos e contextos. 1ed. Fortaleza CE: Universidade Federal do Ceará, p. 286-306.

Melo, R.da S.; Carvalho, M. J. S. (2014) “Aplicativos Educacionais Livres Para Mobile Learning", XI EVIDOSOL e VIII CILTEC-Online - junho/2014 - Disponível em: http://evidosol.textolivre.org. Acesso em: 10 jan/2016.

Mossman, M.; Gomes, L. dos S.; Gluz, J. C. (2012) “Objetos de Aprendizagem Móveis para Ensino de Dedução Natural na Lógica Proposicional," In: Congresso Brasileiro de Informática na Educação, Rio de Janeiro. Anais do Simpósio Brasileiro de Informática na Educação, p. 1-5.

O'malley, C. et al. (2003) "MOBIlearn WP4 - Guidelines for learning/teaching/tutoring in a mobile environment" $\quad<$ http://www.mobilearn.org/download/results/guidelines.pdf $>$. Retrieved February.

Orlandi, B. H.; Isotani, S. (2012) "Uma Ferramenta para Distribuição de Conteúdo Educacional Interativo em Dispositivos Móveis", In: Congresso Brasileiro de Informática na Educação, 2012, Rio de Janeiro. Anais do Simpósio Brasileiro de Informática na Educação, p. 1-5.

Rachid, C. L.; Ishitani, L. (2012) "M-tutorial: ferramenta de autoria para desenvolvimento de tutoriais voltados para o m-learning”, Revista Brasileira de Informática na Educação, Revista, p. 17 - 31, $01 \mathrm{abr} / 2012$.

Saboia, J.; Vargas, P. L. de.; Viva, M. A. de A. (2014) "O uso dos dispositivos móveis no processo de ensino e aprendizagem no meio virtual", Revista cesuca virtual: conhecimento sem fronteiras v.1, n. 1, jul/2013. Disponível em: http://ojs.cesuca.edu.br/index.php/cesucavirtual. Acesso em: 10 jan/2016.

Saccol, A.; Shlemmer, E. ; Barbosa, J. (2011) M-learning e u-learning: Novas perspectiva das aprendizagens móvel e ubíqua. São Paulo: Pearson Prentice Hall.

Santaella, L. (2013) Comunicação ubíqua - repercussões na cultura e na educação. São Paulo: Paulus.

Sharples, Taylor e Vavoula, (2005) "Towards a theory of mobile learning" In: Proceedings of mLearn, v. 1, n. 1, p. 1-9, <http://www.mlearn.org/mlearn2005/CD/papers/SharplesTheoryofMobile.pdf>.

Unesco. (2013) Diretrizes de políticas para a aprendizagem móvel. $<$ http://unesdoc.unesco.org/images/0022/002277/227770por.pdf $>$.

Winter, N. (2006) "What is mobile learning?" In: Sharples, M. (Ed.). Big issues in mobile learning - report of a workshop by the kaleidoscope network of excellence mobile learning initiative. Learning Sciences Research Institute, University of Nottingham, $<$ http://hal.archives-ouvertes.fr/docs/00/19/02/54/PDF/Sharples_Big_Issues.pdf $>$. 